\title{
Inhibitory effect of microRNA-24 on fatty acid- binding protein expression on 3T3-L1 adipocyte differentiation
}

M. Kang ${ }^{1 *}$, L.M. Yan ${ }^{2 *}$, Y.M. Li ${ }^{3}$, W.Y. Zhang ${ }^{3}$, H. Wang ${ }^{3}$, A.Z. Tang ${ }^{1}$ and H.S. Ou ${ }^{3}$

${ }^{1}$ Department of Radiotherapy, First Affiliated Hospital of Guangxi Medical University, Nanning, China ${ }^{2}$ Center of Biochemistry, University of South China, Hengyang, China ${ }^{3}$ College of Pharmacology, Guangxi Medical University, Nanning, China

*These authors contributed equally to this study.

Corresponding authors: H.S. Ou / A.Z. Tang

E-mail: heshengoucn@163.com / tanganzhou@126.com

Genet. Mol. Res. 12 (4): 5267-5277 (2013)

Received August 15, 2013

Accepted March 27, 2013

Published November 7, 2013

DOI http://dx.doi.org/10.4238/2013.November.7.1

ABSTRACT. We examined the effect of microRNAs on 3T3-L1 adipocyte differentiation and expression of adipocyte-specific gene fatty acid-binding protein 4 (FABP4). We screened and identified adipo-related microRNAs during 3T3-L1 adipocyte differentiation with a microRNA microarray. High expression plasmids of miR-24 and miR-21 were constructed and transfected into 3T3-L1 preadipocytes by lipofectamine. The effects of miR-24 and miR-21 on 3T3-L1 adipocyte differentiation were observed, and the protein and mRNA expression levels of FABP4 and AP-1 were determined. The expression profiles of microRNAs significantly changed during 3T3-L1 adipocyte differentiation. The expression of 33 microRNAs was downregulated, among which downregulation of miR-24 was the most extensive. There were 17 microRNAs with upregulated expression; the highest levels were found for miR-21. miR-24 significantly inhibited 3T3-L1 
adipocyte differentiation and maturity, while miR-21 had no significant effect. In addition, miR-24 significantly inhibited the expression of FABP4, while it upregulated AP-1 expression, but had no effect on the level of FABP4 mRNA. miR-21 had no effect on FABP4 protein and mRNA expression. AP-1 silencing could, at least partially, reverse the inhibitory effect of miR-24 on FABP4 expression. We conclude that microRNA expression profiles change significantly during 3T3$\mathrm{L} 1$ adipocyte differentiation and that miR-24 plays an important role in regulating adipocyte differentiation and FABP4 expression. The mechanism involved may be the upregulation of AP-1.

Key words: MicroRNA; 3T3-L1 preadipocyte; FABP4; AP-1; Adipocyte differentiation

\section{INTRODUCTION}

Diabetes is one of the major diseases endangering human health. Clinically, it is divided into 2 major types- type I and type II diabetes. Type II diabetes accounts for the majority of patients with diabetes, and the main manifestation is insulin resistance. Research shows that type II diabetes is positively associated with obesity and that adipocyte differentiation is closely involved in glycolipid metabolism, insulin resistance, and type II diabetes (Briones et al., 2012). At present, the regulatory mechanisms of adipocyte differentiation and their relationship with the occurrence and development of diabetes are not clear.

MicroRNAs (miRNAs) are endogenous small RNAs with a length of 18-24 base pairs (bp). They show a high degree of conservation across species and participate in a variety of vital activities. Previous studies have found that miRNAs play an important role in tumorigenesis (He et al., 2007), cardiovascular diseases (Thum et al., 2008), diabetes (Walker, 2008), and other diseases. miRNAs bind to the 3' untranslated region (UTR) of target mRNAs and regulate their expression either by affecting mRNA stability or by inhibiting translation. miRNAs are intimately involved in development, cell proliferation and differentiation, cell apoptosis and metabolism, and other physiological and pathological processes (Ambros, 2004; Ventura and Jacks, 2009). Some miRNAs are involved in regulating the expression of disease-related genes (Xie et al., 2009; Sun et al., 2009). Recent research suggests that miRNAs may be involved in the pathological processes underlying diabetes (Dehwah et al., 2012). For example, miRNA-143 can promote 3T3-L1 adipocyte differentiation by targeting the growth factor pleiotrophin, a heparin-binding growth factor (Yi et al., 2011). Similarly, miR-22 was shown to inhibit the differentiation of mesenchymal stem cells into adipose tissue and into adipocytes (Huang et al., 2012). These data suggest that different miRNAs have differing effects on adipocyte differentiation. Which miRNAs are involved in adipocyte differentiation and whether they have an effect on the expression of adipocytespecific genes is not clear so far.

In this study, the adipo-specific miRNAs were identified by miRNA microarray analysis. The effect of 2 miRNAs identified by the microarray analysis on adipocyte differentiation was investigated by transfecting them into 3T3-L1 preadipocytes and assaying for changes in the expression of the adipocyte-specific gene fatty-acid binding protein 4 (FABP4). 


\section{MATERIAL AND METHODS}

\section{Materials}

The main materials used were as follows: Dulbecco's Modified Eagle Medium (DMEM, Gibco, USA), Fetal bovine serum (FBS, Tianiin Haoyang Biological Manufacture Co., Ltd., China), Plasmid kit and PCR kit (Tiangen biochemical technology (Beijing) Co., Ltd., China), Total RNA purification Kit (TRIzol) (BioTeke (Beijing) Corporation, China), RTPCR kit [Fermentas(MBI), USA)], Lipofectamine ${ }^{\mathrm{TM}} 2000$ Kit (Invitrogen, USA), Puromycin (Sigma, USA), primer and siRNA synthesis kits (Shanghai Sangon Biological Engineering Technology \& Services Co., Ltd., China), Rabbit anti-FABP4 polyclonal antibody (Cell Signaling Technology, USA), AP-1 monoclonal antibody (sc-166540) (Santa Cruz Biotech, USA), miRNASelect ${ }^{\mathrm{TM}}$ pEGP-miR (Cell Biolabs, USA), mouse 3T3-L1 preadipocytes (Institute of Basic Medical Science, Chinese Academy of Medical Sciences, China), miRCURY ${ }^{\mathrm{TM}}$ Array (Exiqon, Denmark), and Escherichia coli DH5 a (Molecular Medicine Research Center, Institute of Pharmacology, University of South China, China). The sequence of the siRNA used or AP-1 silencing was 5'-AACTGCATGGACCTAACAT-3' (GenBank accession No. NM002228).

\section{Screening of miRNAs associated with adipocyte differentiation}

The screening of miRNAs associated with 3T3-L1 adipocyte differentiation was performed using the miRNA microarray according to the manufacturer protocol. The 3T3-L1 preadipocytes and differentiated adipocytes were collected and total RNA was extracted. After further extraction and labeling labeling with Fluorescent label $\left(\mathrm{Hy}^{\mathrm{TM}}\right)$ in the reaction system (RNA $4.0 \mu \mathrm{L}$, labeling buffer $3.0 \mu \mathrm{L}$, Fluorescent label (Hy3 ${ }^{\mathrm{TM}}$ ) $1.5 \mu \mathrm{L}$, DMSO $2.0 \mu \mathrm{L}$, labeling enzyme $2.0 \mu \mathrm{L}$ ), the concentrated labeled sample was hybridized to the miRNA chips (miRCURY ${ }^{\mathrm{TM}}$ Array). The fluorescence intensity imaging of the microarray and data analysis were performed using relative values as the expression intensity.

\section{Construction of miRNA expression plasmids}

After microarray screening, miR-21 and miR-24, which had differential expression levels in preadipocytes and differentiated adipocytes, were selected for further study. The nucleotide sequences of miR-21 and miR-24 are 5'-UAGCUUAUCAGACUGAUGUUGA-3' and 5'-UGGCUCAGUUCAGCAGGAACAG-3', respectively (http://www.mirbase.org). The sequence of miR-21 and miR-24 were used to design the hairpin miRNA. In brief, a loop of 9 nucleotides (5'-UUCAAGACA-3') was used for the production of miRNAs with $\mathrm{pEP}$-miR vector (Catalog No. MIR-EXP-GP, Cell Biolabs, Inc., San Diego, CA, USA). The stemloop precursor sequence of the miRNA was then added $100 \mathrm{bp}$ native upstream and downstream. Then the desired miRNA precursor (100 bp + stemloop $+100 \mathrm{bp}$ ) was produced by PCR and subsequently cloned into the $B a m \mathrm{HI}$ and NheI sites of the EF-1 intron in the pEP-MiR vector. The plasmids contained a G418 antibiotic selection marker to allow the identification of positively transfected cells. The sequences and orientations of the inserts were verified by sequencing.

\section{Culturing and transfection of 3T3-L1 preadipocytes}

3T3-L1 preadipocytes were cultured in DMEM ( $10 \%$ fetal bovine serum, $1 \times 10^{5} \mathrm{U} / \mathrm{L}$ 
penicillin and $100 \mathrm{mg} / \mathrm{L}$ streptomycin) at $37^{\circ} \mathrm{C}$ temperature, $5 \% \mathrm{CO}_{2}$, and saturated humidity. The Lipofectamine ${ }^{\mathrm{TM}} 2000$ Kit was used for transfecting cells with the miR-21 and miR-24 expression plasmids. Stably transfected clones were identified by puromycin selection $48 \mathrm{~h}$ after transfection.

\section{Differentiation of 3T3-L1 preadipocytes and the identification of differentiated adipocytes}

After 2 days of fusion of 3T3-L1 preadipocytes, the classic cocktail method (Cowherd et al., 1999) was used to induce their differentiation. 3T3-L1 preadipocytes were cultured in DMEM with $0.5 \mathrm{mM}$ Isobuthylxanthine (IBMX), $1 \mu \mathrm{M}$ dexamethasone, $10 \mu \mathrm{g} / \mathrm{mL}$ insulin, and $10 \%$ FBS for $48 \mathrm{~h}$, then in DMEM with $10 \mu \mathrm{g} / \mathrm{mL}$ insulin and $10 \% \mathrm{FBS}$ for another $48 \mathrm{~h}$, and finally they were cultured in DMEM containing 10\% FBS. After 10-12 days of inducing differentiation, the differentiated adipocytes were identified from this heterogeneous population of cells by Oil-red-O staining. Briefly, cell monolayers were washed twice with PBS, fixed with $10 \%$ formalin in PBS (pH 7.4), then washed twice with distilled water. Cells were then stained for $1 \mathrm{~h}$ at room temperature in freshly diluted Oil-Red-O solution. For each dish, three images were captured.

\section{Determination of $F A B P 4$ and $A P-1$ mRNA expression after transfection}

The expression of FABP4 mRNA after transfection was determined by reversetranscriptase polymerase chain reaction (RT-PCR) using the RT-PCR kit [Fermentas (MBI), USA)] according to the manufacturer protocol. The primers for amplifying FABP4 and $\beta$-actin cDNA were designed using the Beacon Designer 2.0 software. The sequence of the upstream primer for $F A B P 4$ was 5'-AAATCACCGCAGACGACA-3' and that of the downstream primer was 5'-CACATTCCACCACCAGCT-3'. For AP-1, the sequence of upstream primer was 5'-CACGTTAACAGTGGGTGCCA-3' and downstream primer, 5'-CCCCGACGGTCTCTC TTCA-3'. For $\beta$-actin, the upstream and downstream primer sequences were 5'-GTCCCTCA CCCTCCCAAAAG-3' and 5'-GCTGCCTCAACACCTCAACCC-3', respectively, and the length of product was $266 \mathrm{bp}$. cDNA was synthesized using the RevertAid ${ }^{\mathrm{TM}}$ first strand cDNA synthesis kit. The RT-PCR amplification was performed using cDNA as the template according to the standard protocol. Initial denaturation: $95^{\circ} \mathrm{C}$ for $2 \mathrm{~min} ; 30$ cycles: Denature at $95^{\circ} \mathrm{C}$ for $30 \mathrm{~s}$; Anneal at $55^{\circ} \mathrm{C}$ for $30 \mathrm{~s}$; Extend at $72^{\circ} \mathrm{C}$ for $45 \mathrm{~s}$. After amplification, agarose gel electrophoresis was conducted on $2 \%$ agarose gel with $5 \mu \mathrm{L}$ PCR products.

\section{Determination of FABP4 and AP-1 protein expression after transfection}

The expression of FABP4 protein after transfection was determined by Western blotting. Differentiated 3T3-L1 adipocytes were treated with protein cleavage enzyme. After centrifugation, the supernatant protein was obtained, and $30 \mu \mathrm{L}$ supernatant protein was run on an $8 \%$ sodium dodecylsulfate polyacrylamide gel electrophoresis (SDS-PAGE) gel. The protein was then transferred to a polyvinylidene fluoride (PVDF) membrane, which was blocked with skim milk powder for $1 \mathrm{~h}$. After being incubated with primary antibody overnight, the membrane was washed with Tris-buffered saline Tween-20 (TBST) 3 times for $10 \mathrm{~min}$ each. Then, 
horseradish peroxidase conjugated secondary antibody was added. After incubation for $1 \mathrm{~h}$, the membrane was washed and developed using the aqueous solutions A and B, which are from the Western Blot kit (Invitrogen, USA). The protein was visualized by X-ray film exposure.

\section{Statistical analysis}

Data are reported as means \pm standard deviation (SD). Statistical analysis was performed using the SPSS 12.0 statistical software (SPSS, USA). P $<0.05$ was considered statistically significant.

\section{RESULTS}

\section{Changes in the miRNA expression profile during adipocyte differentiation}

As shown in Tables 1 and 2, 33 miRNAs were found to be downregulated (miR-24, miR-22, miR-296, etc.) whereas 17 miRNAs were found to be upregulated (miR-143, miR21 , miR-199, miR-142, etc.) in differentiated adipocytes as compared to preadipocytes. This indicates that the expression profiles of miRNAs changes during adipocyte differentiation.

\begin{tabular}{|c|c|c|}
\hline microRNAs & Fold-change & $\mathrm{P}$ \\
\hline mmu-miR-24 & -3.89 & 0.0007 \\
\hline mmu-miR-296 & -3.87 & 0.0009 \\
\hline mmu-miR-15a & -3.79 & 0.0137 \\
\hline mmu-miR-15b & -3.74 & 0.0215 \\
\hline mmu-miR-16 & -3.39 & 0.0046 \\
\hline mmu-miR-17-3p & -3.27 & 0.0049 \\
\hline mmu-miR-25 & -2.98 & 0.0328 \\
\hline mmu-miR-26a & -2.87 & 0.0437 \\
\hline mmu-miR-26b & -2.70 & 0.0294 \\
\hline Uknown_mmu target & -2.62 & 0.0159 \\
\hline mmu-miR-29a & -2.56 & 0.0157 \\
\hline mmu-miR-10a & -2.41 & 0.0173 \\
\hline Unknown mmu target & -2.19 & 0.0359 \\
\hline mmu-miR-292-5p & -2.17 & 0.0367 \\
\hline mmu-let-7f & -2.15 & 0.0104 \\
\hline Unknown mmu target & -2.13 & 0.0107 \\
\hline mmu-let-7d & -2.09 & 0.0327 \\
\hline mmu-miR-292-5p MM2 & -2.05 & 0.0432 \\
\hline mmu-miR-182 & -1.97 & 0.0281 \\
\hline mmu-let-7a & -1.95 & 0.0445 \\
\hline mmu-miR-10b & -1.93 & 0.0154 \\
\hline mmu-miR-23b & -1.90 & 0.0289 \\
\hline mmu-miR-320 & -1.87 & 0.0425 \\
\hline mmu-miR-23a & -1.85 & 0.0373 \\
\hline mmu-let-7e & -1.75 & 0.0159 \\
\hline mmu-miR-16 & -1.71 & 0.0432 \\
\hline mmu-miR-22 & -1.48 & 0.0185 \\
\hline mmu-miR-33 & -1.45 & 0.0071 \\
\hline mmu-miR-292-3p & -1.42 & 0.0033 \\
\hline Unknown mmu target & -1.40 & 0.0059 \\
\hline mmu-let-7c & -1.30 & 0.0099 \\
\hline mmu-miR-298 & -1.31 & 0.0102 \\
\hline mmu-let-7b & -1.28 & 0.0105 \\
\hline
\end{tabular}


Table 2. Upregulated microRNAs during 3T3-L1 adipocyte differentiation.

\begin{tabular}{lcr}
\hline microRNAs & Fold-change & P \\
\hline Unknown mmu target & 1.07 & 0.0022 \\
mmu-miR-27a & 1.23 & 0.0087 \\
mmu-miR-291a-5p & 1.36 & 0.0465 \\
mmu-miR-143 & 1.65 & 0.0453 \\
mmu-let-7g & 1.77 & 0.0451 \\
Unknown mmu_target & 1.92 & 0.0266 \\
mmu-miR-29a & 1.99 & 0.0481 \\
mmu-miR-189 & 2.08 & 0.0427 \\
Unknown mmu target & 2.17 & 0.0004 \\
mmu-miR-146a & 2.29 & 0.0352 \\
Unknown mmu target & 2.51 & 0.0169 \\
mmu-miR-27b & 2.68 & 0.0173 \\
Unknow mmu target & 2.76 & 0.0293 \\
mmu-miR-146b & 2.89 & 0.0327 \\
mmu-miR-199b & 3.29 & 0.0042 \\
mmu-miR-142 & 3.55 & 0.0153 \\
mmu-miR-21 & 3.89 & 0.0012 \\
\hline
\end{tabular}

\section{Effect of miR-21 and miR-24 on adipocyte differentiation}

After transfecting preadipocytes with miR-21 and miR-24, they were differentiated into adipocytes, which were identified by Oil-red-O staining. Morphological changes in the 3T3-L1 adipocytes after transfection were observed. The results are shown in Figure 1. Mature cells with red lipid droplets could be seen in untransfected 3T3-L1 adipocytes and in 3T3-L1 adipocytes transfected with miR-21. However, there were no red lipid droplets in 3T3-L1 adipocytes transfected with miR-24 although the cellular morphology of these cells was different from that of fused 3T3-L1 preadipocytes.

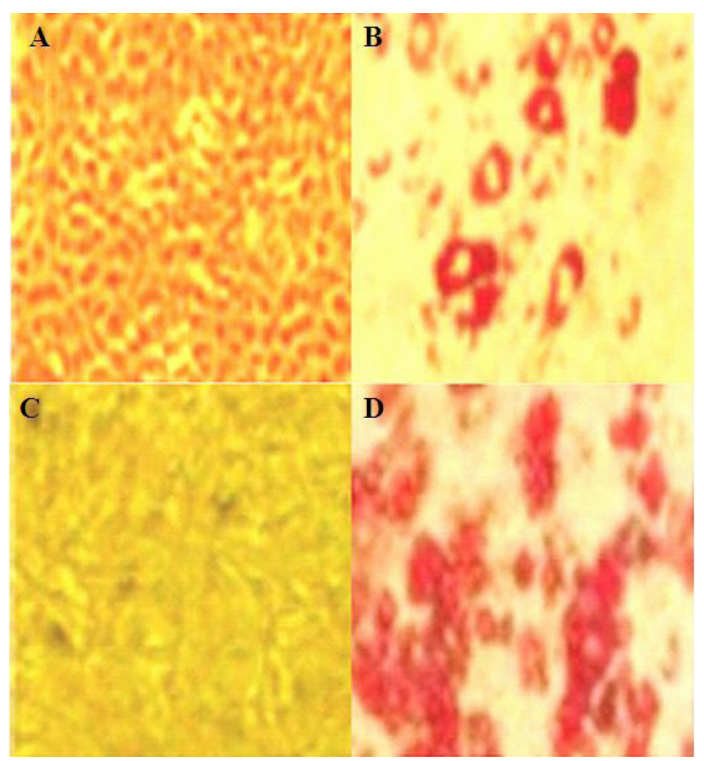

Figure 1. Oil-red-O stained 3T3-L1 cells after differentiation (40X). A. 3T3-L1 preadipocytes; B. 3T3-L1 adipocytes; C. 3T3-L1 adipocytes transfected with miR-24; D. 3T3-L1 adipocytes transfected with miR-21. 


\section{Effects of miR-21 and miR-24 on FABP4 mRNA and protein expression}

As shown in Figure 2, FABP4 mRNA was not expressed in differentiated 3T3-L1 adipocytes. There was no apparent change in FABP4 mRNA expression levels in 3T3-L1 adipocytes transfected with either miR-24 or miR-21. The effect of miR-21 and miR-24 on FABP4 protein expression is shown in Figure 3. There was no FABP4 protein expression in 3T3-L1 preadipocytes. After differentiation, the FABP4 protein expression level in 3T3-L1 adipocytes transfected with miR-21 was not significantly different from that of untransfected 3T3-L1 adipocytes. However, the FABP4 protein expression level in 3T3-L1 adipocytes transfected with miR-24 was significantly lower than in untransfected cells.
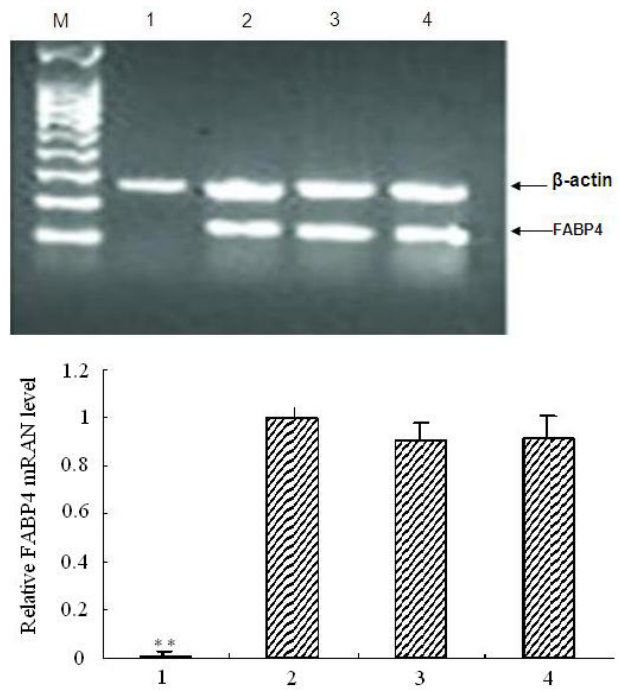

Figure 2. Effect of miR-21 and miR-24 overexpression on FABP4 mRNA expression. Lane $M=$ marker; lane $1=$ 3T3-L1 preadipocytes; lane $2=3 \mathrm{~T} 3-\mathrm{L} 1$ adipocytes; lane $3=3 \mathrm{~T} 3-\mathrm{L} 1$ adipocytes transfected with miR-24; lane $4=$ 3T3-L1 adipocyte transfected with miR-21. **P $<0.01,1$ vs 2, 3, and 4.
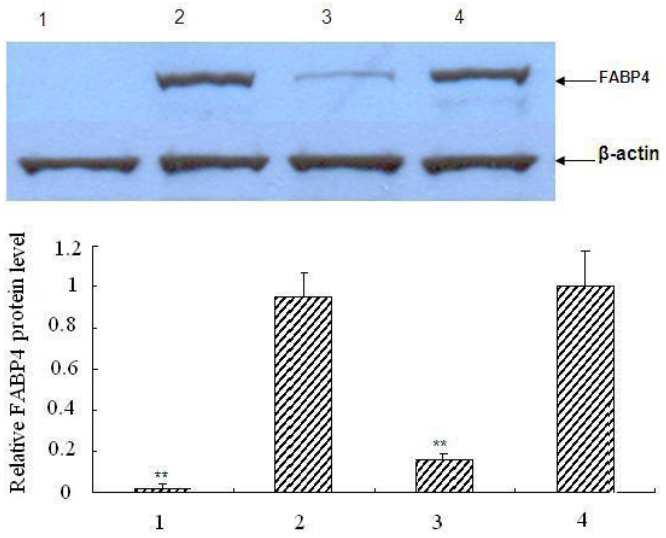

Figure 3. Effect of miR-21 and miR-24 overexpression on FABP4 protein expression. Lane $1=3 \mathrm{~T} 3-\mathrm{L} 1$ preadipocytes; lane $2=3 \mathrm{~T} 3-\mathrm{L} 1$ adipocytes; lane $3=3 \mathrm{~T} 3-\mathrm{L} 1$ adipocytes transfected with miR-24; lane $4=3 \mathrm{~T} 3-\mathrm{L} 1$ adipocytes transfected with miR-21. $* * \mathrm{P}<0.01,3$ vs 2. 


\section{Reversal of the miR-24 inhibitory effect by AP-1 siRNA}

As shown by the above results, miR-24 overexpression significantly decreased FABP4 expression. The effect of miR-24 overexpression on AP-1 mRNA and protein expression was also determined. The results are shown in Figures 4 and 5. AP-1 mRNA and protein expression levels were significantly higher in 3T3-L1 adipocytes transfected with miR-24 than in untransfected adipocytes. This indicates that miR-24 overexpression can significantly up-regulate AP-1 mRNA and protein expression levels. We also investigated the effect of AP-1 silencing on the miR-24 induced change in FABP4 expression. The results are shown in Figure 6. The FABP4 protein level in 3T3-L1 adipocytes transfected with miR-24 and AP-1 siRNA was lower than that of untransfected adipocytes, but higher than that of 3T3-L1 adipocytes transfected with only miR-24. As shown in Figures 2 and 3, miR-24 significantly inhibited FABP4 mRNA and protein expression. Therefore, we concluded that AP-1 gene silencing could, at least partially, reverse the inhibitory effect of miR-24 on FABP4 protein expression.

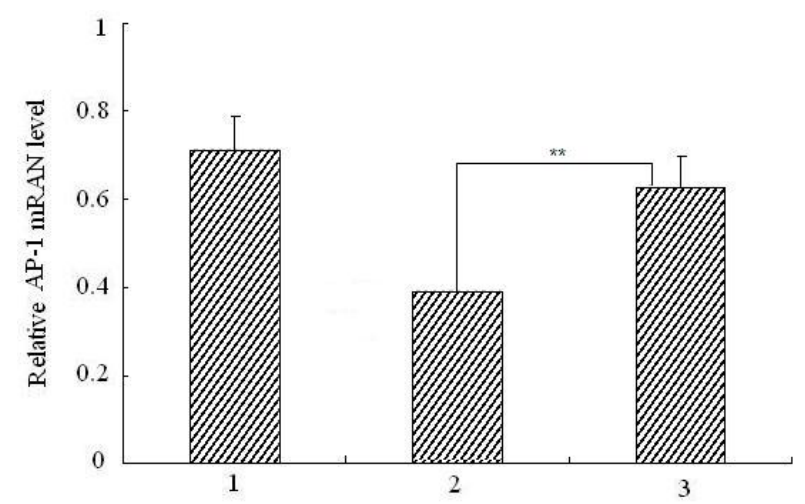

Figure 4. Effect of miR-24 on AP-1 mRNA expression. Column $1=3 \mathrm{~T} 3-\mathrm{L} 1$ preadipocytes; Column $2=3 \mathrm{~T} 3-\mathrm{L} 1$ adipocytes transfected with miR-24; Column $3=$ untransfected 3T3-L1 adipocytes. **P $<0.01,2$ vs 3.
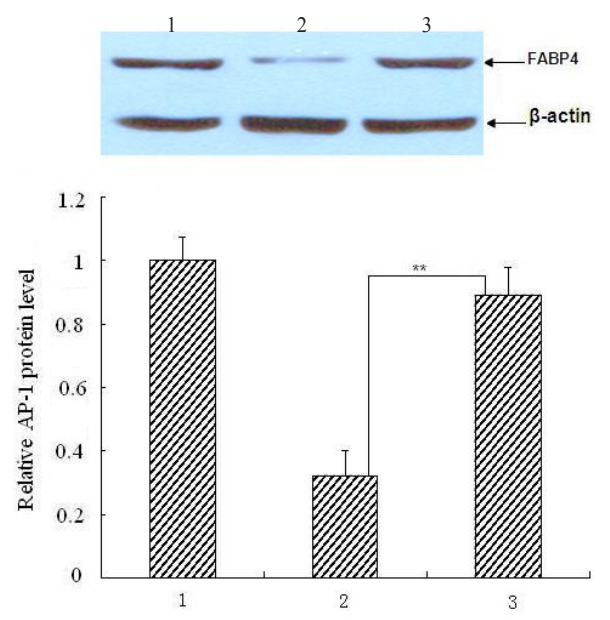

Figure 5. Effect of miR-24 on AP-1 protein expression. Lane 1 = 3T3-L1 preadipocytes; lane 2 = 3T3-L1 adipocytes transfected with miR-24; lane $3=$ untransfected 3T3-L1 adipocytes. $* * \mathrm{P}<0.01,2$ vs 3. 


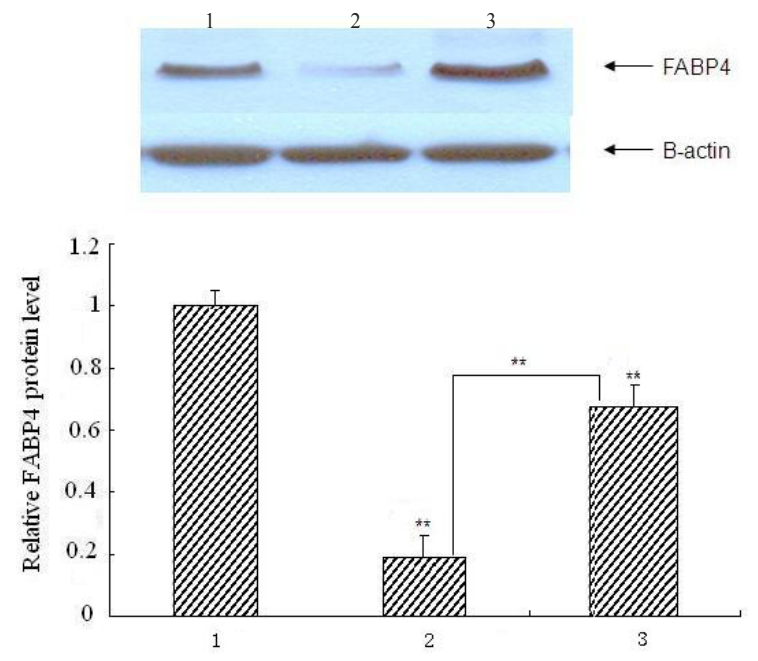

Figure 6. Effect of AP-1 silencing on the inhibitory effect of miR-24 on FABP4 protein expression. Lane 1=3T3L1 adipocytes; lane $2=3 \mathrm{~T} 3-\mathrm{L} 1$ adipocytes transfected with miR-24; lane 3 $=3 \mathrm{~T} 3$-L1 adipocytes transfected with miR-24 plasmid and AP-1 siRNA. **P $<0.01,2$ vs 3.

\section{DISCUSSION}

The main purpose of our study was to explore the effect of miRNAs on adipocyte differentiation and adipocyte-specific gene expression. Adipo-related miRNAs were identified and transfected into 3T3-L1 preadipocytes. The molecular mechanism of the miRNA regulation of adipocyte differentiation and the effect of this regulation on the expression of adipocytespecific genes are discussed. Our results show that the expression profiles of miRNAs change significantly during 3T3-L1 preadipocyte differentiation, and that miR-24 overexpression can significantly inhibit preadipocyte differentiation, and decrease the expression of FABP4. This suggests that miRNAs play an important role in regulating adipocyte differentiation and adipocyte-specific gene expression. The role of miRNAs on adipocyte differentiation and gene expression has also been demonstrated by earlier studies. As reported by Xie et al. (2009) the ectopic expression of miR-103 and miR-143 in preadipocytes can accelerate adipocyte formation. Wang et al. (2008) found that the up-regulation of miR-17-92 can accelerate adipocyte differentiation and triglyceride accumulation. In addition, the expression of miR335 is upregulated in the adipose tissues of mice with obesity, and this increased expression may be related to the pathophysiological mechanism of obesity (Nakanishi et al., 2009).

Another important finding of this study is that miR-24 overexpression can significantly inhibit $F A B P 4$ expression. To our knowledge, our study is the first to report an inhibitory effect of miR-21 on FABP4. FABP4 is expressed mainly in adipocytes and macrophages (Xu et al., 2003). Its expression and activity increase during adipocyte differentiation and promote adipocyte differentiation. In addition, FABP4 plays a key role in obesity-induced insulin resistance (Hotamisligil et al., 1996). Studies in a Fabp4 knockout mouse model showed that Fabp 4 plays a key role in multiple metabolic syndromes, including diabetes and atherosclerosis. Furuhashi et al. (2007) found that a small-molecule inhibitor of FABP4 is effective against atherosclerosis and type II diabetes in an animal model. FABP4 regulates the expression of key 
enzymes and its receptor in lipid metabolism. The FABP4 protein participates in intracellular signal transduction and plays an important role in lipid metabolism and transport (Tang et al., 2004). In this study, we found that the overexpression of miR-24 significantly inhibits the expression of FABP4 protein but not FABP 4 mRNA in adipocytes. This suggests that miR-24 regulates FABP4 protein expression by inhibiting the translation of FABP4 mRNA, eventually affecting adipocyte differentiation. FABP4 promotes adipocyte differentiation (Yang et al., 1989; Urs et al., 2006). In this study, the inhibition of FABP4 protein expression by miR24 overexpression adversely affected adipocyte differentiation. Therefore, this suggests that miR-24 may regulate adipocyte differentiation by reducing FABP4 protein levels. Interestingly, our study finds that miR-24 can up-regulate the expression of AP-1. AP-1 is a dimeric complex of the Jun and Fos proteins. As a transcription factor, it is involved in the regulation of the physiological and pathophysiological processes involved in many diseases. At present, the role of AP-1 in adipocyte differentiation is not clear. It was found that the activation of JNK-Jun can prevent lipogenesis by PPAR- $\gamma$ phosphorylation (Hu et al., 1996; Adelmant et al., 1998) and can inhibit the differentiation of mesenchymal stem cells into adipocytes by down-regulating CREB (Tominaga et al., 2005). This study also finds that AP-1 expression increases after 3T3-L1 preadipocytes differentiate into adipocytes. While miR-24 overexpression inhibits FABP4 expression, AP-1 expression increases significantly upon miR-24 overexpression. In addition, AP-1 silencing using siRNAs can reverse the inhibitory effect of miR-24 on FABP4. This may be due to the collaborative regulation of FABP 4 by miR-24 and AP-1. This study may be the first to report this phenomenon. Whether there are other transcription factors involved in the miR-24-induced inhibition of adipocyte differentiation and FABP 4 expression remains to be studied.

\section{ACKNOWLEDGMENTS}

Research supported by the National Natural Science Foundation of China (\#81373403) and the Research Foundation of the Education Department of Guangxi Province, China (\#2013YB049).

\section{REFERENCES}

Adelmant G, Gilbert JD and Freytag SO (1998). Human translocation liposarcoma-CCAAT/enhancer binding protein (C/ EBP) homologous protein (TLS-CHOP) oncoprotein prevents adipocyte differentiation by directly interfering with C/EBP $\beta$ function. J. Biol. Chem. 273: 15574-15581.

Ambros V (2004). The functions of animal microRNAs. Nature 431: 350-355.

Briones AM, Nguyen Dinh CA, Callera GE, Yogi A, et al. (2012). Adipocytes produce aldosterone through calcineurindependent signaling pathways: implications in diabetes mellitus-associated obesity and vascular dysfunction. Hypertension 59: 1069-1078.

Cowherd RM, Lyle RE and McGehee RE Jr (1999). Molecular regulation of adipocyte differentiation. Semin. Cell Dev. Biol. 10: 3-10.

Dehwah MA, Xu A and Huang Q (2012). MicroRNAs and type 2 diabetes/obesity. J. Genet. Genomics 39: 11-18.

Furuhashi M, Tuncman G, Gorgun CZ, Makowski L, et al. (2007). Treatment of diabetes and atherosclerosis by inhibiting fatty-acid-binding protein aP2. Nature 447: 959-965.

He L, He X, Lim LP, de Stanchina E, et al. (2007). A microRNA component of the p53 tumour suppressor network. Nature 447: 1130-1134.

Hotamisligil GS, Johnson RS, Distel RJ, Ellis R, et al. (1996). Uncoupling of obesity from insulin resistance through a targeted mutation in aP2, the adipocyte fatty acid binding protein. Science 274: 1377-1379. 
Hu E, Kim JB, Sarraf P and Spiegelman BM (1996). Inhibition of adipogenesis through MAP kinase-mediated phosphorylation of PPARgamma. Science 274: 2100-2103.

Huang S, Wang S, Bian C, Yang Z, et al. (2012). Upregulation of miR-22 promotes osteogenic differentiation and inhibits adipogenic differentiation of human adipose tissue-derived mesenchymal stem cells by repressing HDAC6 protein expression. Stem Cells Dev. 21: 2531-2540.

Nakanishi N, Nakagawa Y, Tokushige N, Aoki N, et al. (2009). The up-regulation of microRNA-335 is associated with lipid metabolism in liver and white adipose tissue of genetically obese mice. Biochem. Biophys. Res. Commun. 385 : 492-496.

Sun T, Fu M, Bookout AL, Kliewer SA, et al. (2009). MicroRNA let-7 regulates 3T3-L1 adipogenesis. Mol. Endocrinol. 23: 925-931.

Tang QQ, Zhang JW and Daniel LM (2004). Sequential gene promoter interactions of C/EBP $\beta$, C/EBP $\alpha$, and PPARgamma during adipogenesis. Biochem. Biophys. Res. Commun. 319: 235-239.

Thum T, Gross C, Fiedler J, Fischer T, et al. (2008). MicroRNA-21 contributes to myocardial disease by stimulating MAP kinase signalling in fibroblasts. Nature 456: 980-984.

Tominaga S, Yamaguchi T, Takahashi S, Hirose F, et al. (2005). Negative regulation of adipogenesis from human mesenchymal stem cells by Jun N-terminal kinase. Biochem. Biophys. Res. Commun. 326: 499-504.

Urs S, Harrington A, Liaw L and Small D (2006). Selective expression of an aP2/Fatty Acid Binding Protein 4-Cre transgene in non-adipogenic tissues during embryonic development. Transgenic Res. 15: 647-653.

Ventura A and Jacks T (2009). MicroRNAs and cancer: short RNAs go a long way. Cell 136: 586-591.

Walker MD (2008). Role of MicroRNA in pancreatic B-cells: where more is less. Diabetes 57: 2567-2568.

Wang Q, Li YC, Wang J, Kong J, et al. (2008). miR-17-92 cluster accelerates adipocyte differentiation by negatively regulating tumor-suppressor Rb2/p130. Proc. Natl. Acad. Sci. U. S. A. 105: 2889-2894.

Xie H, Lim B and Lodish HF (2009). MicroRNAs induced during adipogenesis that accelerate fat cell development are downregulated in obesity. Diabetes 58: 1050-1057.

Xu Y, Iyengar S, Roberts RL, Shappell SB, et al. (2003). Primary culture model of peroxisome proliferator-activated receptor gamma activity in prostate cancer cells. J. Cell. Physiol. 196: 131-143.

Yang VW, Christy RJ, Cook JS, Kelly TJ, et al. (1989). Mechanism of regulation of the 422(aP2) gene by cAMP during preadipocyte differentiation. Proc. Natl. Acad. Sci. U. S. A. 86: 3629-3633.

Yi C, Xie WD, Li F, Lv Q, et al. (2011). MiR-143 enhances adipogenic differentiation of 3T3-L1 cells through targeting the coding region of mouse pleiotrophin. FEBS Lett. 585: 3303-3309. 